\title{
Pitting Fault Detection of a Wind Turbine Gearbox Using Empirical Mode Decomposition
}

\author{
Wei Teng $1,2,{ }^{*}$ - Feng Wang ${ }^{1}$ - Kaili Zhang 1 - Yibing Liu ${ }^{1}$ - Xian Ding 1 \\ ${ }^{1}$ North China Electric Power University, School of Energy Power and Mechanical Engineering, China \\ 2 Taiyuan University of Technology, Key Laboratory of Advanced Transducers and Intelligent Control Systems, \\ Ministry of Education, China
}

\begin{abstract}
The conventional method of detecting a gear fault is to demodulate the vibration signal collected from the gearbox based on the Hilbert transform; however, this requires human intervention and lacks sophistication. Empirical mode decomposition (EMD) is a significant timefrequency tool for adaptively decomposing vibration signals into a collection of intrinsic mode functions (IMFs); a fault feature can be extracted from one of IMFs to reveal the fault location and fault level of a gear or bearing in the mechanical drive system. In this paper, a multi-harmonic vibration model of a gearbox with fault modulation is presented, a conventional demodulation analysis using Hilbert transform is introduced, and the principle of EMD is illustrated. The Hilbert demodulation analysis and EMD are applied to processing field vibration signals collected from a wind turbine gearbox to detect a gear-pitting fault. The results show that EMD can extract the fault modulation information more adaptively and intelligently than Hilbert demodulation analysis can.
\end{abstract}

Keywords: empirical mode decomposition, adaptively, fault detection, wind turbine, gearbox

\section{INTRODUCTION}

In recent years, wind energy has had great development due to the ongoing need for renewable energy. At the same time, the failure rate of key components in wind turbines has greatly increased because of severe operational environments. The wind turbine gearbox is one of the most fragile components in the turbine because of extreme differences in temperature and complicated alternating loads from wind turbulence. In many wind farms throughout the world, gearboxes fail only several thousand hours after wind turbine is put into operation, and its service life is much less than designed. For example, between 2000 and 2004 in Sweden the downtime resulting from gearbox failure was longer than that from other components [1]. In China, the bearings and gears in wind turbines can easily be destroyed because the wind load is unbalanced and irregular with regard to time [2]. Wind turbines will shut down if gearboxes fail, with consequential inevitable economic losses. Therefore, finding a fault feature-extracting method of wind turbine gearbox under a complicated operating environment remains an urgent task, as does building a maintenance mechanism with high efficiency that can guarantee wind turbine and power grid safety.

Many new techniques for fault feature extraction have emerged for rotating machine health diagnosis. For example, Wang et al. combined a complex wavelet transform-based envelope extraction of speed-varying vibration signals with computed order tracking to eliminate speed dependence in the sampled data [3], which showed the effectiveness in identifying bearing structural defects under varying operating conditions. Li et al. adopted an adaptive stochastic resonance to extract the fault feature of machine accuracy decay in boring and milling machines [4]. Significant motor features, such as bearing failure, broken rotor bar, phase unbalance etc. from vibration signals are extracted through the scale-invariant feature transform algorithm to generate the faulty symptoms [5]. Generally, the structure of a wind turbine gearbox is complex and diverse, consisting of multistage planetary gears and ordinary gears. Therefore, many signal-processing methods were applied to different types of gearboxes in order to find existing or potential faults. Barszcz and Randall [6] detected a tooth crack in the planetary gear of a wind turbine using spectral kurtosis. Yang et al. [7] used continuous wavelet transform to diagnose gearboxes in wind turbine test rig. Tang et al. [8] studied Morlet wavelet transformation and Wigner-Ville distribution, and detected a simulant groove on a wind turbine gearbox test-bed. A discrete wavelet transform is applied to the demodulated current signal of an induction motor to detect a multistage gearbox fault by removing the intervening neighbouring features [9] and [10]. The previous studies accumulated rich experience in fault diagnosis of wind turbines. However, affected by random excitation of variable rotational speed and alternating load, vibration signals from wind turbine gearbox are non-stationary or nonlinear, so conventional signal processing methods, such as Fourier transform and wavelet analysis et al., lack processing ability for these types of signals. Empirical mode decomposition (EMD) was presented 
by Huang et al. [11] to adaptively decompose a nonstationary signal into a collection of intrinsic mode functions (IMFs); EMD has been widely applied to measurement analysis and fault diagnosis in mechanical transmission. For example, Loutridis [12] constructed a variable stiffness dynamic model of a single-stage gear transmission, and employed the energy of the second IMF from EMD as the fault feature to denote the gear crack depth. Liu et al. [13] developed a B-spline EMD as a filter bank to analyse the vibration signals from automobile gearbox, which showed that the Hilbert spectrum was more effective than continuous wavelet transform in the detection of the vibration signatures. Fast Fourier transform of IMF from EMD was utilized to detect bearing faults [14]. Yu et al. [15] and Cheng et al. [16] used time-frequency entropy from Hilbert-Huang to accurately identify gear status with or without fault; they proposed a local Hilbert energy spectrum to provide energy distribution based on EMD to extract the characteristic information of a gear fault. A merit index was introduced by Ricci and Pennacchi [17] to select IMF automatically, and the defective gearbox was always identified univocally by using the merit index. Planetary gearbox faults can be found by matching the dominant peaks in the envelope spectrum and the spectrum of instantaneous frequency with the theoretical characteristic frequencies of faulty gears through the joint application of the amplitude and frequency demodulation methods [18]. Zhang and Zhou [19] used ensemble empirical mode decomposition and an optimized support vector machine to realize multi-fault diagnosis in rolling element bearings.

The abovementioned research shows that EMD can adequately process non-stationary vibration signals. However, these successful cases are mostly based on simulation signals or test rigs, which are significantly different from actual situations. There are few applications on field vibration signals, and the effectiveness of EMD for field data cannot be validated, especially for wind turbine gearboxes under variable rotational speed and alternating load.

This paper is organized as follows: a vibration model of a gearbox with fault modulation and a conventional demodulation analysis using the Hilbert transform are discussed in Section 1. In Section 2, the principle of EMD is presented. In Section 3, the structure and parameters of an actual wind turbine gearbox are introduced, the feature frequencies of the gearbox are calculated, and the field vibration signals are analysed to detect a gear pitting fault based on EMD and Hilbert demodulation, respectively.
In addition, the fault cause of wind turbine gearbox is also discussed in this section. The conclusions are given in Section 4.

\section{VIBRATION SIGNAL OF GEARBOX AND DEMODULATION ANALYSIS}

\subsection{Vibration Signal of Gearbox}

During gear mesh in the gearbox, the excitation due to variable stiffness generates vibration signal consisting of typical multi-harmonic components. The signal above mentioned can be described as [20]:

$$
x(t)=\sum_{m=1}^{M} x_{m} \cos \left(2 \pi f_{z} m t+\varphi_{m}\right),
$$

where $x(t)$ denotes the vibration time signal of gearbox, $x_{m}$ denotes the amplitude of the $m$ order harmonic, $\varphi_{m}$ denotes the phase of the $m$ order harmonic, and $f_{z}$ denotes the gear mesh frequency, which is also a carrier frequency.

When a defect happens in a gear system, there will be a multi-component amplitude modulation phenomenon in the gearbox vibration signal. The modulation function can be shown as:

$$
a_{m}(t)=\sum_{n=1}^{N} A_{m, n} \cos \left(2 \pi f_{n} n t+\alpha_{m, n}\right),
$$

where $a_{m}(t)$ denotes a amplitude modulation function, $A_{m, n}$ denotes the amplitude of the $n$ order harmonic of the modulation function, $\alpha_{m, n}$ denotes the phase of the $n$ order harmonic of the modulation function, and $f_{n}$ denotes the rotational frequency of the shaft fixed with defective gear, which is also a modulation frequency.

From Eqs. (1) and (2), the model of gearbox vibration signal with fault modulation can be shown as:

$$
x(t)=\sum_{m=1}^{M} x_{m}\left[1+a_{m}(t)\right] \cos \left(2 \pi f_{z} m t+\varphi_{m}\right)
$$

\subsection{Hilbert Demodulation Analysis}

The Hilbert transform is an attractive method for demodulation of a vibration signal with fault modulation. For a gearbox vibration signal consisting of multiple harmonics and noise, the key step of demodulation analysis using the Hilbert transform is to separate it using a band-pass filter. Neglecting the phase information, the vibration signal in Eq. (3) after band-pass filtering can be processed as:

$$
x_{m}(t)=x_{m}\left[1+A_{m, x} \cos \left(2 \pi f_{x} t\right)\right] \cos \left(2 \pi f_{z} m t\right),
$$


where $A_{m, x}$ denotes the amplitude of a certain order harmonic of the modulation function, and $f_{x}$ denotes a certain modulation frequency.

Applying the Hilbert transform [21] and [22] to Eq. (4), the following is obtained:

$$
\begin{aligned}
\hat{x}_{m}(t) & =x_{m}(t) * \frac{1}{\pi t}=\frac{1}{\pi} \int_{-\infty}^{\infty} \frac{x_{m}(\tau)}{t-\tau} d \tau= \\
& =x_{m}\left[1+A_{m, x} \cos \left(2 \pi f_{x} t\right)\right] \sin \left(2 \pi f_{z} m t\right) .
\end{aligned}
$$

An analytic expression is given as:

$$
z_{m}(t)=x_{m}(t)+j \hat{x}_{m}(t)
$$

Then the envelope of $x_{m}(t)$ is shown as:

$$
\begin{aligned}
\left|z_{m}(t)\right| & =\sqrt{x_{m}^{2}(t)+\hat{x}_{m}^{2}(t)}= \\
& =x_{m}\left|1+A_{m, x} \cos \left(2 \pi f_{x} t\right)\right| .
\end{aligned}
$$

The envelope in Eq. (7) is the amplitude of modulation signal in some frequency band and the corresponding phase is described as:

$$
\theta(t)=\arctan \frac{\hat{x}_{m}(t)}{x_{m}(t)}=2 \pi f_{z} m t .
$$

\section{EMPIRICAL MODE DECOMPOSITION}

Empirical mode decomposition is an effective timefrequency tool for processing non-stationary or nonlinear vibration signals, which can decompose a signal into a collection of intrinsic mode functions (IMFs), each of which can be linear or nonlinear. There are two conditions that each IMF must satisfy [11]: a) the number of extrema (maxima and minima) must be equal to the number of zero crossing points or differ at most by one; b) at any point, the mean value of the envelope defined by the local maxima and the one defined by the local minima is zero.

The procedure of EMD can be described as:

1) All the local extrema in the raw signal $x(t)$ are sought out and the maxima should be connected using a cubic spline line, and the same with the local minima. Then, the upper and lower envelopes arise, as well as the mean value $m(t)$ of the above envelopes.

2) Subtracting the mean envelope $m(t)$ from $x(t)$, $h_{1}(t)$ can be shown as:

$$
h_{1}(t)=x(t)-m(t) .
$$

If $h_{1}(t)$ cannot satisfy the two conditions of IMF above, it will be treated as a raw signal similar to $x(t)$ and steps 1) and 2) will be repeated until $h_{1}(t)$ becomes one IMF, which can be described as:

$$
c_{1}(t)=h_{1}(t) \text {. }
$$

3) Subtracting the first IMF $c_{1}(t)$ from $x(t)$, the remaining signal $r_{1}(t)$ can be shown as:

$$
r_{1}(t)=x(t)-c_{1}(t)
$$

4) Then, treating $r_{1}(t)$ as a raw signal similar to $x(t)$, and the second, the third until the $n$ order IMF can be calculated by repeating the above steps, which can be noted as $c_{2}(t), c_{3}(t), \ldots, c_{n}(t)$. The iterative process will not end until it satisfies some criterion, and the last residual signal is $r_{n}(t)$. Thus, the primary raw signal $x(t)$ can be decomposed into a collection of IMFs and a residual signal, which is shown as:

$$
x(t)=\sum_{i=1}^{n} c_{i}(t)+r_{n}(t)
$$

Then, after applying the Hilbert transform to each IMF, the result is

$$
\hat{c}_{i}(t)=\frac{1}{\pi} \int_{-\infty}^{\infty} \frac{c_{i}(\tau)}{t-\tau} d \tau
$$

The analytical function $z_{i}(t)$ is calculated as:

$$
z_{i}(t)=c_{i}(t)+j \cdot \hat{c}_{i}(t)=a_{i}(t) \cdot e^{j \phi_{i}(t)} .
$$

where the amplitude function, i.e. the envelope of $c_{i}(t)$, is

$$
a_{i}(t)=\sqrt{c_{i}(t)^{2}+\hat{c}_{i}(t)^{2}} .
$$

The phase function can be described as:

$$
\phi_{i}(t)=\arctan \frac{\hat{c}_{i}(t)}{c_{i}(t)} .
$$

Then the instantaneous frequency of each IMF is shown as:

$$
\omega_{i}(t)=\frac{d \phi_{i}(t)}{d t}
$$

\section{TESTING AND ANALYSIS OF WIND TURBINE GEARBOX}

In a wind farm in Inner Mongolia, China, maintenance technicians observed intense noise in a wind turbine gearbox when making a routine inspection. Therefore, vibration testing was carried out on this fixed-pitch and fixed-speed wind turbine, whose nominal output power was $600 \mathrm{~kW}$. 
Although force measurement with simple signal processing showed the effectiveness in identifying the fault rating and fault type of bearing [23], it cannot be applied to the sealed wind turbine gearbox, because there are no spaces provided to install force transducers. In this case, acceleration measurement was adopted; this is commonly used to monitor and diagnose gear faults. The acceleration transducer is based on piezoelectric effect, with a dynamic response range from 0.1 to $10000 \mathrm{~Hz}$, a resonance frequency about $30000 \mathrm{~Hz}$, and a sensitivity of $500 \mathrm{mV} / \mathrm{g}$.

Theoretically, the acceleration transducers should be installed on the location with maximum casing deformation to obtain excellent testing performance, however, modal analysis for casing is necessary; this will enhance computational complexity. Therefore, setting aside the question of the best transducer location, we glued four acceleration transducers on the casing of the gearbox from low- to high-speed stages, which are shown as Figs. 1a and b. The vibration signals from acceleration transducers are transferred to data acquisition system and then to a host computer through Ethernet. The data acquisition system is shown in Figs. 1c and d; it has a 14-bit analogue-todigital converter. In order to obtain a wide frequency band, the sample frequency is $16384 \mathrm{~Hz}$, and the cutoff frequency of the anti-aliasing filter is $7000 \mathrm{~Hz}$.
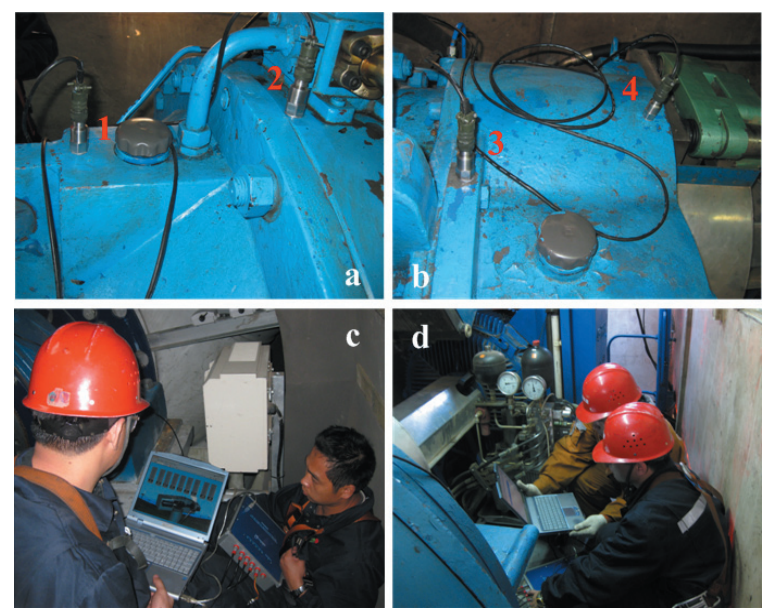

Fig. 1. Test system on field wind turbine gearbox; a) two transducers in the front of the gearbox, b) two transducers in the rear of the gearbox, c) data acquisition system, d) data analysis and processing

\subsection{Structure of Wind Turbine Gearbox}

A wind turbine gearbox is a device that converts the low rotational speed of turbine's rotor into the high speed of generator. The structure of the tested wind turbine gearbox is shown in Fig. 2; it consists of a onestage planetary gear and two-stage ordinary gears.

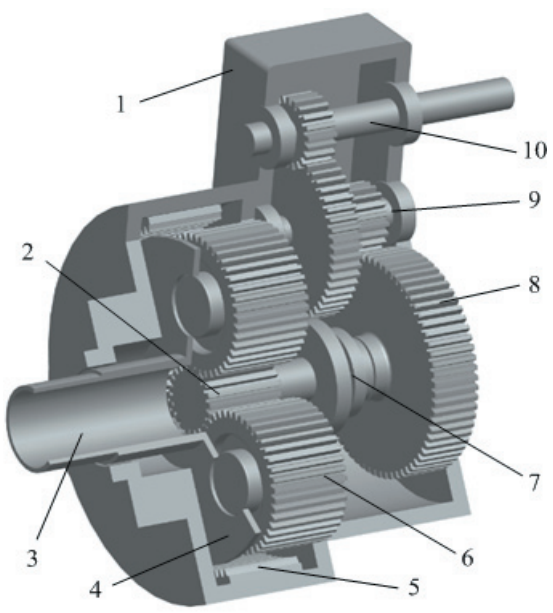

Fig. 2. Structure of wind turbine gearbox

1-casing, 2-sun gear, 3-turbine's rotor, 4-planetary arm, 5-ring gear, 6-planetry gear; there are a total of three planets in all, 7-sun shaft, 8-wheel, 9-middle shaft, and 10-high-speed shaft

The multistage transmissions in a wind turbine gearbox can be divided into three parts, which can be seen as the planetary stage, middle stage and highspeed stage in Fig. 3. Among which, $Z_{s}$ denotes the number of sun gear teeth, $Z_{p}$ denotes the number of planet teeth, $Z_{c}$ denotes the number of ring gear teeth, $Z_{m i}$ denotes the number of drive gear teeth in the middle stage, $Z_{m o}$ denotes the number of non-drive gear teeth in the middle stage, $Z_{h i}$ denotes the number of drive gear teeth in the high-speed stage and $Z_{h o}$ denotes the number of non-drive gear teeth in the highspeed stage. From Fig. 3, it can be determined that the rotational speed of the planetary arm in the gearbox is equal to that of the turbine's rotor, that the planetary gear is driven by the arm and meshed with the sun gear and the ring gear, and the sun gear and wheel (8) in Fig. 2 are concentric. The mechanical power generated from turbine rotation is first transferred to the sun shaft through the planetary stage, then to the middle shaft through the middle stage, and finally to the high-speed shaft through the gear mesh of the high-speed stage, which is connected with the shaft of generator.

The numbers of teeth in wind turbine gearbox are listed in Table 1.

Table 1. The numbers of teeth of multiple gears in wind turbine gearbox

\begin{tabular}{ccccccc}
\hline$Z_{p}$ & $Z_{s}$ & $Z_{c}$ & $Z_{m i}$ & $Z_{m 0}$ & $Z_{h i}$ & $Z_{\text {ho }}$ \\
\hline 43 & 21 & 117 & 68 & 20 & 54 & 21 \\
\hline
\end{tabular}




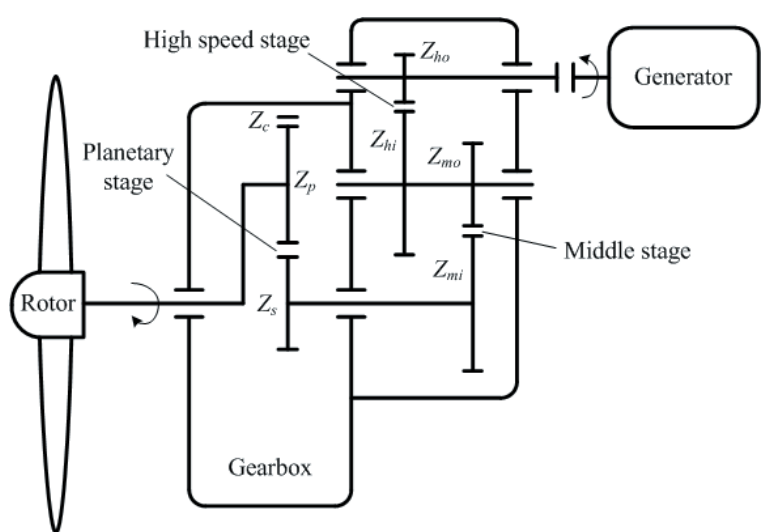

Fig. 3. Definition of multistage transmissions in wind turbine gearbox

The rotational speed of turbine's rotor is 26.8 $\mathrm{rpm}$, and the total transmission ratio of the gearbox is 56.56. The relative feature frequencies of vibration signal from the gearbox are calculated as follows: the shaft rotational frequency is:

$$
f_{n}=n / 60 .
$$

The mesh frequency of ordinary gear is:

$$
f_{z}=n Z / 60
$$

and the mesh frequency of planetary gear is:

$$
f_{z}=n_{a} Z_{c} / 60,
$$

where $n$ denotes a certain shaft's rotational speed whose unit is rpm, $Z$ denotes the number of the ordinary gear teeth, $n_{a}$ denotes the rotational speed of the planetary arm, and $Z_{c}$ denotes the number of the ring gear teeth.

From Eqs. (18) to (20), it is possible to calculate multistage shaft rotational frequencies and gear mesh frequencies in the wind turbine gearbox, shown in Table 2.

Table 2. Shaft rotational frequencies, gear mesh frequencies

\begin{tabular}{lc}
\hline \multicolumn{1}{c}{ Rotational frequency } & Frequency $[\mathrm{Hz}]$ \\
\hline Rotor & 0.447 \\
\hline Sun shaft & 2.9 \\
\hline Middle shaft & 9.8 \\
\hline High-speed shaft & 25.3 \\
\hline \multicolumn{1}{c}{ Mesh frequency } & Frequency [Hz] \\
\hline Planetary stage & 52.32 \\
\hline Middle stage & 196.39 \\
\hline High-speed stage & 530.25 \\
\hline
\end{tabular}

\subsection{Hilbert Demodulation Analysis}

Gear crack or pitting in a gearbox leads to a modulation phenomenon, which takes the gear mesh frequency, gear natural frequency, or casing natural frequency as a carrier frequency, and takes the rotational frequency of the shaft fixed with defective gear as the modulation frequency. In order to identify the defective gear, Hilbert demodulation analysis is adopted. At first, a modulation signal is separated from the raw signal through a band-pass filter; next, the modulation signal is processed using the Hilbert transform to obtain the modulation frequencies that are characteristic of the fault.

The time vibration signals of four acceleration transducers are shown in Fig. 4. The vibration amplitude of first transducer fixed on the planetary stage of the gearbox is shown in Fig. 4a, which is obviously smaller than other transducers due to the low rotational speed of the turbine's rotor. From the time signals in Fig. 4, little fault information about the gearbox can be determined. Consequently, the time signals in the frequency domain are converted using the Fourier transform, and the results of the second transducer are shown in Fig. 5. The linear power spectrum density (PSD) in Fig. 5b and the logarithmic power spectrum density in Fig. $5 \mathrm{c}$ both represent the mesh frequency $(195 \mathrm{~Hz})$ of the middle stage and its harmonics, which show greater vibration energy in the middle stage than others. For the obvious modulation phenomenon in Fig. 5c (shown as enclosed in ellipse),
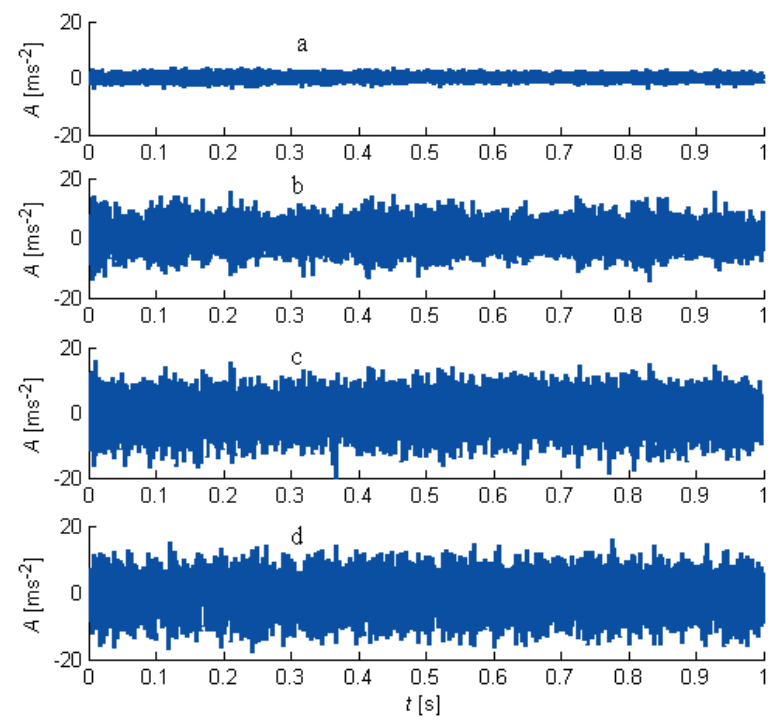

Fig. 4. Time vibration signals from the four acceleration transducers; a) the first, b) the second, c) the third and d) the fourth 

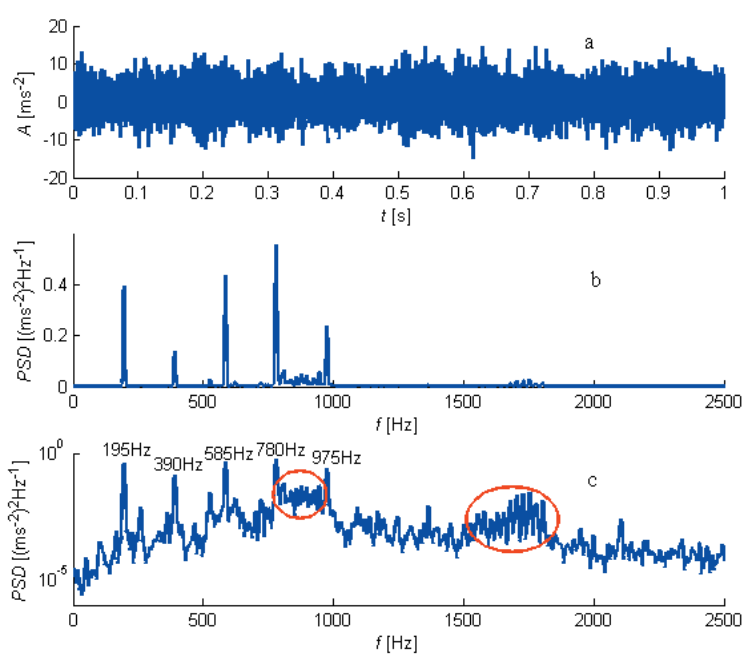

Fig. 5. Time signal from the second transducer and its PSD; a) time signal, b) linear power spectrum density and c) logarithmic power spectrum density
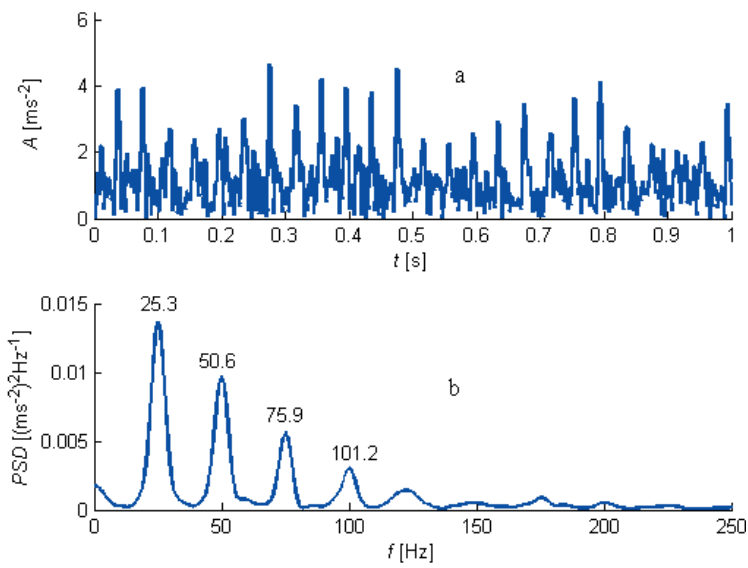

Fig. 6. The results after band-pass filtering with cut-off frequency 1500 and $1800 \mathrm{~Hz}$; a) envelope of filtered time signal, and b) envelope spectrum
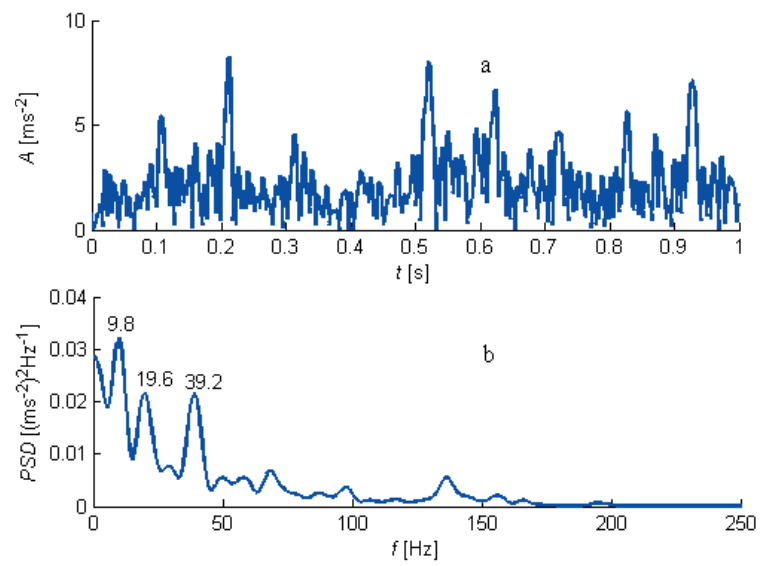

Fig. 7. The results after band-pass filtering with cut-off frequency 820 and $972 \mathrm{~Hz}$; a) envelope of filtered time signal, and b) envelope spectrum two 6-order Butterworth band-pass filters are used to process the time signals, and the cut-off frequencies are $820,972,1500$ and $1800 \mathrm{~Hz}$, respectively. Next, the Hilbert transform is used to analyse the filtered time signals, and the envelopes with corresponding PSD are shown in Figs. 6 and 7, respectively.

Fig. $6 \mathrm{~b}$ distinctly shows the rotational frequency of the high-speed shaft and its harmonics. The analogous result arises in Fig.7b, in which the humps represent the rotational frequency of the middle shaft and its harmonics. The demodulated frequencies (rotational frequency of the high speed and middle shaft) and harmonics in Figs. 6 and 7 show that the gears in the high-speed stage can be faulty or even fail.

\subsection{Vibration Analysis Based on EMD}

Next, the vibration signal is decomposed from the second transducer using EMD without the modulation frequency band (enclosed in ellipse) considered in Fig. 5c. The decomposition process is carried out according to Eqs. (9) through (17). The first four IMFs are listed in Fig. 8, and the corresponding envelope spectra of the IMFs are shown in Fig. 9. The rotational frequency of the high-speed shaft and its harmonics are visible in IMF1, as is the rotational frequency of the middle shaft in IMF2, which shows that EMD can extract the modulation frequency automatically without pre-processing as with Hilbert demodulation analysis.

Considering occasionality in the above single group of vibration signal, 57 groups are gathered each minute over the course of about one hour and processed based on EMD. The span of each group signal is one second. The waterfall map of the first IMF's envelope spectrum is shown in Fig. 10. The rotational frequency of the high-speed shaft and its harmonics are clearly visible throughout, which confirms that the defective gear exists in the highspeed stage. In Fig. 10, the amplitudes of the feature frequencies fluctuate distinctly along groups axis due to the varying speed and load of stochastic wind in one hour, while the feature frequencies almost remain unchanged along the frequency axis because of the characteristics of fixed pitch and fixed speed of the wind turbine. For future study, a quantitative index denoting the fault level of the defective gear for wind turbine gearbox under varying speed and alternating load will be a significant issue.

Fifty-seven groups of vibration signal are analysed using the Hilbert demodulation; the results are shown as in Fig. 11. In the first 15 minutes, the wind speed 

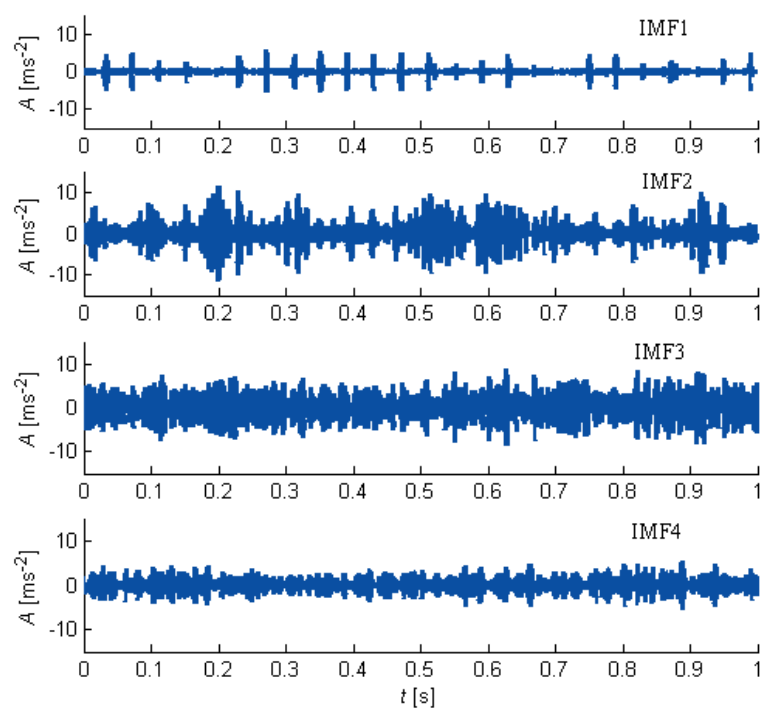

Fig. 8. The first four IMFs of vibration signal from the second transducer
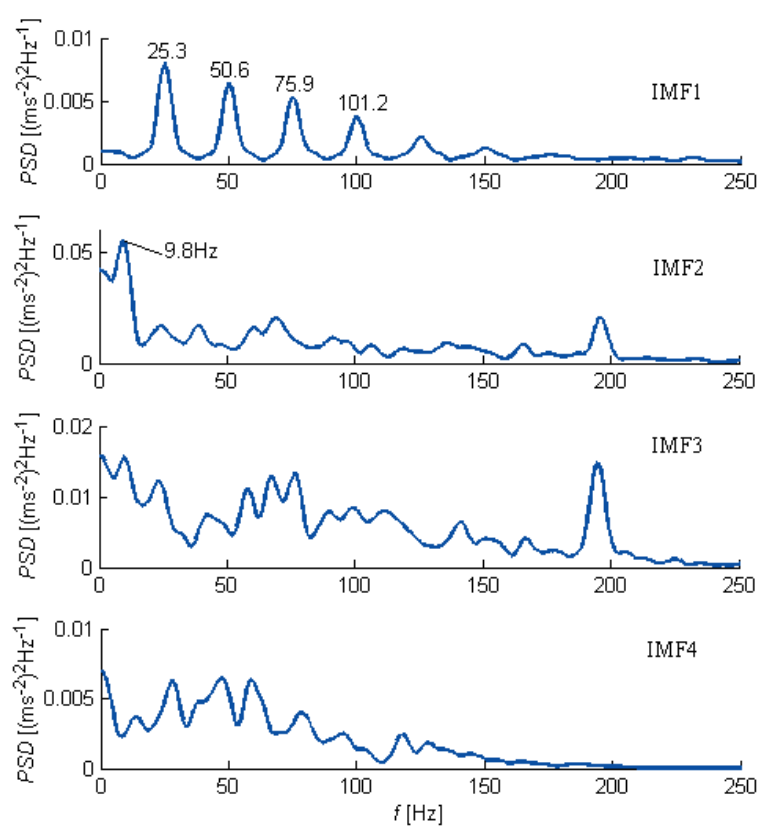

Fig. 9. The envelope spectra of the first four IMFs of vibration signal from the second transducer

is 8.5 to $10.7 \mathrm{~m} / \mathrm{s}$, which generates lager excitation energy than speeds of 5.5 to $6.3 \mathrm{~m} / \mathrm{s}$ in the following 42 minutes. Although the wind speed is varied, the rotational speed of wind turbine remains steady at about $26.8 \mathrm{r} / \mathrm{min}$ due to the stalling characteristics of fixed pitch blades. The lager excitation energy in the first 15 minutes caused the resonance range (modulation frequency band) of the gearbox to deviate from 1500 to $1800 \mathrm{~Hz}$. Therefore, for the first 15 groups, the multi-harmonic components of high

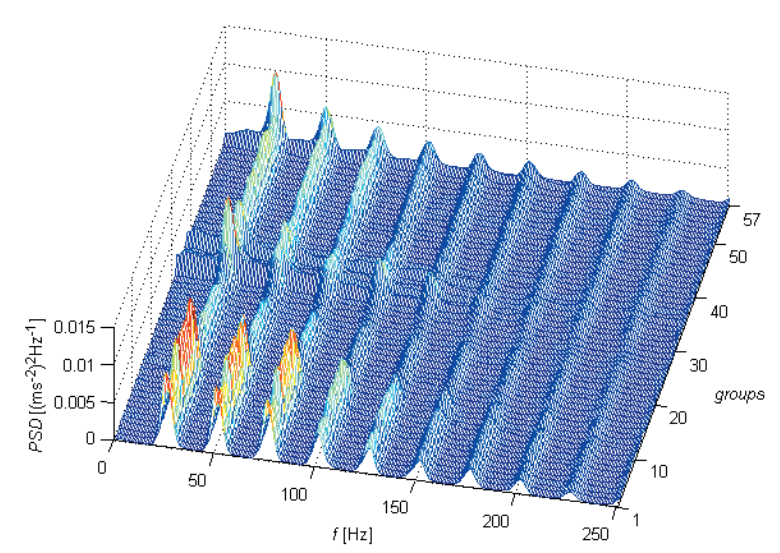

Fig. 10. Waterfall map of the first IMFs after EMD from 57 groups of vibration signal

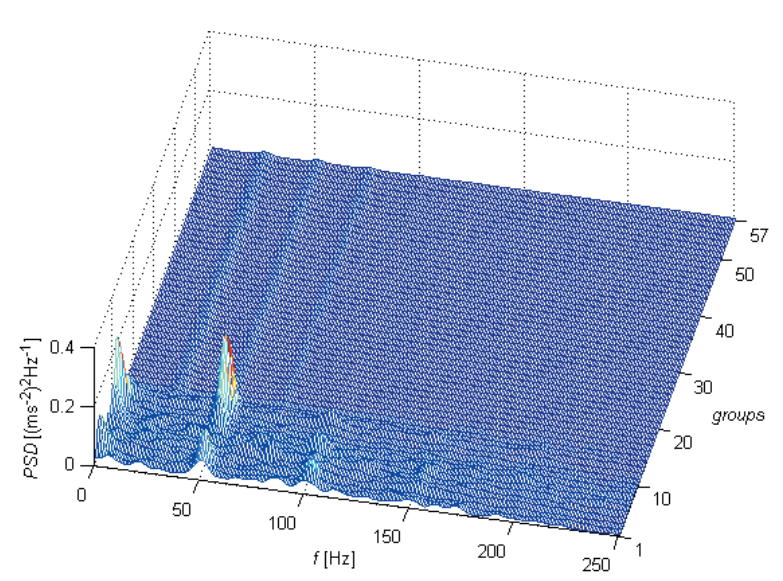

Fig. 11. Waterfall map of the 57 groups of vibration signal using Hilbert demodulation with the cut-off frequency 1500 and $1800 \mathrm{~Hz}$ of band-pass filter

speed shaft cannot be demodulated effectively using the band-pass filter with cut-off frequencies 1500 and $1800 \mathrm{~Hz}$. In contrast, due to the resonance range between 1500 and $1800 \mathrm{~Hz}$ in the later 42 minutes, the Hilbert demodulation can still obtain multi-harmonic components of the high-speed shaft. Compared with the Hilbert demodulation analysis as shown in Fig.11, EMD is more adaptive to obtain better demodulation effect in Fig. 10, especially for the first 15 groups. To be precise, the cut-off frequency of the band-pass filter in the Hilbert demodulation requires human selection and it is difficult to obtain the optimal value. EMD can extract the fault feature frequency more adaptively, and be independent of the selection of the modulation frequency band.

Only the vibration signal of the second transducer is studied above; the other three ones should be discussed. Next, the vibration signals from all the four acceleration transducers are analysed using EMD. 
The first IMFs and corresponding envelope spectra are shown in Fig. 12. Due to the larger distance from the first transducer location to the high-speed stage gear pair, the envelope spectrum in Fig. 12a has no fault features about $25.3 \mathrm{~Hz}$ and its harmonics. In Figs. $12 \mathrm{~b}$ and d, there are components of $25.3 \mathrm{~Hz}$ and its harmonics distinctly in the second transducer and the forth transducer. As for the third transducer in Fig. 12c, the envelope spectrum of the first IMF represents fewer fault features than Figs. $12 \mathrm{~b}$ and d. The reason may be that the third transducer is installed on the ribbed slab of the casing where it is not overly sensitive to the excitation of the high-speed stage.
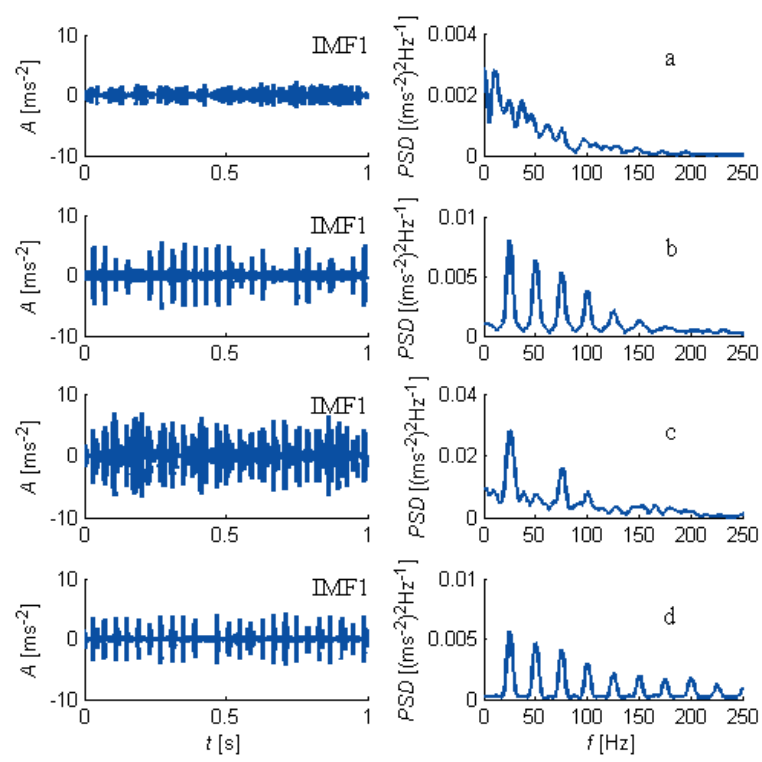

Fig. 12. The first IMFs and their envelope spectra from the four transducers; a) the first, b) the second, c) the third, and d) the forth

\subsection{Discussion about Fault Cause}

Observing the inner condition from the peephole of the wind turbine gearbox, we determined that the two gears of the high-speed stage were seriously affected by pitting, shown as in Fig. 13. After consultation with field technicians, a reason was determined. Because the effect of thermal expansion was not considered when assembling the high-speed shaft of the gearbox with the shaft of generator, an inherent error arose due to the high temperature and high speed during the actual operation of gearbox. Therefore, the high-speed shaft of gearbox was off-centre or bent, which can cause pitting and wear in the gears at the high-speed stage.

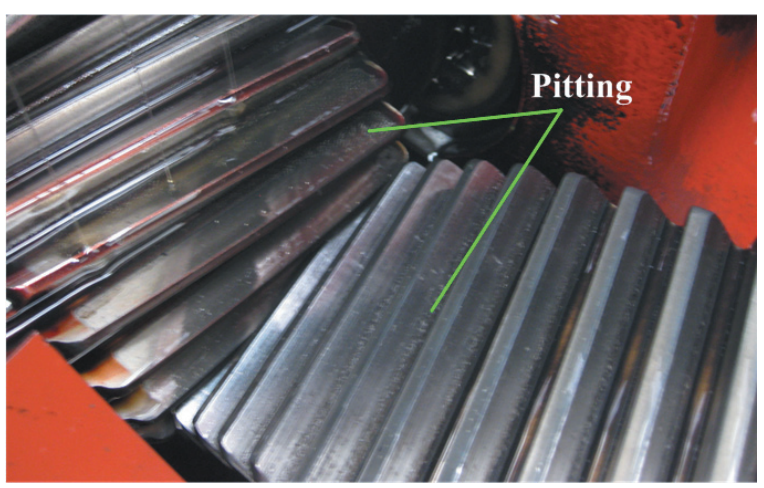

Fig. 13. Pitting in high-speed stage of wind turbine gearbox

\section{CONCLUSION}

The Hilbert demodulation analysis is an effective tool for detecting faults in a wind turbine gearbox; however, this method relies on human intervention to select the modulation frequency band of the bandpass filter. An empirical mode decomposition can decompose the vibration signal of field gearbox and extract the fault feature frequency from an intrinsic mode function adaptively. In this paper, EMD is successfully applied to detect gear pitting faults in a wind turbine gearbox. Due to its better adaptability, EMD can be intelligently used as a powerful tool of fault diagnosis in the wind turbine gearbox.

\section{ACKNOWLEDGEMENTS}

The research presented in this paper was supported by National Natural Science Foundation of China (No. 51305135), the Fundamental Research Funds for the Central Universities of China (No. 12MS06) and the Key Lab of Advanced Transducers and Intelligent Control System (Taiyuan University of Technology), Ministry of Education, Taiyuan, China (No. 201305).

\section{REFERENCES}

[1] Ribrant, J., Bertling, L.M. (2007). Survey of failures in wind power systems with focus on Swedish wind power plants during 1997-2005. IEEE Transactions on Energy Conversion, vol. 22, no. 1, p. 167-173, DOI:10.1109/ TEC.2006.889614.

[2] Liu, W.Y., Tang, B.P., Jiang, Y.H. (2010). Status and problems of wind turbine structural health monitoring techniques in China. Renewable Energy, vol. 35, no. 7, p. 1414-1418, DOI:10.1016/j.renene.2010.01.006.

[3] Wang, J.J., Gao, R.X., Yan, R.Q. (2013). Multiscale enveloping order spectrogram for rotating machine health diagnosis. Mechanical Systems 
and Signal Processing, in Press, DOI:10.1016/j. ymssp.2013.06.001.

[4] Li, B., Li, J.M., Tan, J.Y. (2012). AdSR based fault diagnosis for three-axis boring and milling machine. Strojniski vestnik - Journal of Mechanical Engineering. vol. 58, no. 9, p. 527-533, DOI:10.5545/ sv-jme.2011.272.

[5] Do, V.T., Chong, U.P. (2011). Signal model-based fault detection and diagnosis for induction motors using features of vibration signal in two-dimension domain. Strojniski vestnik - Journal of Mechanical Engineering. vol. 57, no. 9, p. 655-666, DOI:10.5545/ sv-jme.2010.162.

[6] Barszcz, T., Randall, R.B. (2009). Application of spectral kurtosis for detection of a tooth crack in the planetary gear of a wind turbine. Mechanical Systems and Signal Processing, vol. 23, no. 4, p. 1352-1365, DOI:10.1016/j.ymssp.2008.07.019.

[7] Yang, W., Tavner, P.J., Wilkinson, M.R. (2009). Condition monitoring and fault diagnosis of a wind turbine synchoronous generator drive train. IET Renewable Power Generation, vol. 3, no. 1, p. 1-11, DOI:10.1049/iet-rpg:20080006.

[8] Tang, B.P., Liu, W.Y., Song, T. (2010). Wind turbine fault diagnosis based on Morlet wavelet transformation and Wigner-Ville distribution. Renewable Energy, vol. 35, no. 12 , p. 2862-2866, DOI:10.1016/j. renene.2010.05.012.

[9] Mohanty, A.R., Kar, C. (2006). Fault detection in a multistage gearbox by demodulation of motor current waveform. IEEE transactions on Industrial Electronics, vol. 53, no. 4, p. 1285-1297, DOI:10.1109/ TIE.2006.878303.

[10] Amirat, Y., Benbouzid, M.E.H., Al-Ahmar, E., Bensaker B., Turri S. (2009). A brief status on condition monitoring and fault diagnosis in wind energy conversion systems. Renewable and Sustainable Energy Reviews, vol. 13, no. 9, p. 2629-2636, DOI:10.1016/j. rser.2009.06.031.

[11] Huang, N.E., Shen, Z., Long, S.R. (1998). The empirical mode decomposition and the Hilbert spectrum for nonlinear and non-stationary time series analysis. Proceedings of the Royal Society of London Series, vol. 454, p. 903-995, DOI:10.1098/rspa.1998.0193.

[12] Loutridis, S.J. (2004). Damage detection in gear systems using empirical mode decomposition. Engineering Structures, vol. 26, no. 12, p. 1833-1841, DOI:10.1016/j.engstruct.2004.07.007.

[13] Liu, B., Riemenschneider, S., Xu, Y. (2006). Gearbox fault diagnosis using empirical mode decomposition and Hilbert spectrum. Mechanical Systems and Signal
Processing, vol. 20, no. 3, p. 718-734, DOI:10.1016/j. ymssp.2005.02.003.

[14] Rai, V.K., Mohanty, A.R. (2007). Bearing fault diagnosis using FFT of intrinsic mode functions in Hilbert-Huang transform. Mechanical Systems and Signal Processing, vol. 21, no. 6, p. 2607-2615, DOI:10.1016/j.ymssp.2006.12.004.

[15] Yu, D.J., Yang, Y., Cheng, J.S. (2007). Application of time-frequency entropy method based on HilbertHuang transform to gear fault diagnosis. Measurement, vol. 40, no. 9-10, p. 823-830, DOI:10.1016/j. measurement.2007.03.004.

[16] Cheng, J.S., Yu, D.J., Tang, J.S. (2008). Application of frequency family separation method based upon EMD and local Hilbert energy spectrum method to gear fault diagnosis. Mechanism and Machine Theory, vol. 43, no. 6, p. 712-723, DOI:10.1016/j. mechmachtheory.2007.05.007.

[17] Ricci, R., Pennacchi, P. (2011). Diagnostics of gear faults based on EMD and automatic selection of intrinsic mode functions. Mechanical Systems and Signal Processing, vol. 25, no. 3, p. 821-838, DOI:10.1016/j.ymssp.2010.10.002.

[18] Feng, Z.P., Zuo, M.J., Qu, J.(2013). Joint amplitude and frequency demodulation analysis based on local mean decomposition for fault diagnosis of planetary gearboxes. Mechanical Systems and Signal Processing, vol. 40, no. 1, p. 56-75, DOI:10.1016/j. ymssp.2013.05.016.

[19] Zhang, X.Y., Zhou, J.Z. (2013). Multi-fault diagnosis for rolling element bearings based on ensemble empirical mode decomposition and optimized support vector machines. Mechanical Systems and Signal Processing, vol. 41, no. 1-2, p. 127-140, DOI:10.1016/j. ymssp.2013.07.006.

[20] McFadden, P.D. (1986). Detection fatigue cracks in gears by amplitude and phase demodulation of meshing vibration. Journal of Vibration and Acoustics, vol. 108, no. 4, p. 165-170, DOI:10.1115/1.3269317.

[21] Fan, X.F., Zuo, M.J. (2006). Gearbox fault detection using Hilbert and wavelet packet transform. Mechanical Systems and Signal Processing, vol. 20, no. 4, p. 966982, DOI:10.1016/j.ymssp.2005.08.032.

[22] Feldman, M. (2011). Hilbert transform in vibration analysis. Mechanical Systems and Signal Processing, vol. 25, no. 3, p. 735-802, DOI:10.1016/j. ymssp.2010.07.018.

[23] Slavic, J., Brkovic, A., Boltezar, M. (2011). Typical bearing-fault rating using force measurementsapplication to real data. Journal of Vibration and Control, vol. 17, no. 14, p. 2164-2174, DOI: $10.1177 / 1077546311399949$. 